\title{
The effects of peripheral movement and flicker on the detection thresholds of sinusoidal gratings
}

\author{
ALISON BOWLING \\ Monash University, Clayton, Victoria, Australia
}

\begin{abstract}
Contrast sensitivity functions were obtained in the presence of temporally modulated surrounds. Sensitivity to low spatial frequencies was depressed below that found with a steady surround when the surround was either a sinusoidally flickering field or a drifting vertical square-wave grating. This effect was observed both with 1 -sec presentations of 5 - and $0.5-\mathrm{Hz}$ counterphase flicker and with 60-msec pulsed gratings. In addition, reaction time histograms became more characteristic of sustained mechanisms in the presence of temporal modulation. The data were considered in terms of the activity of a nonlinear network of retinal subunits.
\end{abstract}

Kruger $(1980,1981)$ has demonstrated that the behavior of retinal ganglion cells is influenced by the presence of movement in the peripheral visual field. A global pattern was moved over a large portion of a cat's retina excluding the receptive field of the ganglion cell under investigation. The response of an $X$ cell was similar with both steady and moving surrounds, but a Y cell behaved like an $X$ cell when the surrounding pattern was moving. In particular, the nonlinear response to an alternating bipartite field was markedly reduced, and the response to a central spot of light lost its transient nature, becoming more sustained. Consequently, peripheral movement tended to cause $\mathrm{Y}$ cells to behave more like $\mathrm{X}$ cells, and a "transient" time-course to become more "sustained."

Since the shift effect was eliminated in $\mathrm{Y}$ cells in the presence of peripheral movement, it was proposed that the effects of peripheral movement were due to the same mechanism that produces the shift and periphery effects. These effects show properties similar to those of the subunits postulated by Hochstein and Shapley (1976) to account for Y cell nonlinearity, and it has been suggested that the same mechanism is responsible for both (Derrington, Lennie, \& Wright, 1979). It is thus possible that the important difference between $\mathrm{X}$ and $\mathrm{Y}$ cells is that $\mathrm{Y}$ cells are connected to these nonlinear pathways, whereas $X$ cells are not (Kruger, 1980). Alternatively, they may be affected differently by the nonlinear network (Shapley \& Victor, 1978). The sustained or transient behavior of retinal ganglion cells may consequently be determined to a large extent by the degree to which this network is activated.

This experimental work was made possible by the award of an Australian Research Grants Service grant to the author. In addition, the patient endurance of Richard Kasperczyk and Warren Harrison in serving as subjects is gratefully acknowledged. I also thank Ross Day for his suggestions and comments on the manuscript.

The author's mailing address is: Psychology Department, Monash University, Clayton, Victoria, Australia.
The following experiments were concerned with the possibility that there was a psychophysical correlate of the neurophysiological effects of peripheral movement on $X$ and $Y$ cells. A psychophysical parallel to the shift effect had already been demonstrated (Valberg \& Breitmeyer, 1980). Since peripheral movement causes cells with a transient time-course to become more sustained, human mechanisms responding with a transient timecourse might similarly become more sustained in the presence of peripheral movement. The visual response to low spatial frequencies has been shown to be transient in nature, with pronounced on and off peaks (e.g., Mitov, Vassilev, \& Manahilov, 1981; Tolhurst, 1975), and the visual system is also more sensitive to low spatial frequencies when gratings are moving or flickering (e.g., Kulikowski \& Tolhurst, 1973; Robson, 1966). There is, thus, evidence to show that channels sensitive to low spatial frequencies are more sensitive than higher frequency channels to temporal modulation. However, this does not necessarily mean that the difference between the timecourses of the responses to low- and high-frequency gratings indicates the existence of independent "transient" and "sustained" channels, and such a dichotomy will not be assumed here.

Mechanisms responsive to low spatial frequencies respond best when the gratings are moving or flickering, since they respond to the onsets and offsets of stimuli rather than to their prolonged presence. Changing the shape of the response time-course by means of peripheral movement would thus eliminate the advantage of flickering over steady stimuli. in stimulating mechanisms responsive to low spatial frequencies. A decline in sensitivity to counterphase flickering or moving low spatial frequency gratings would therefore be expected to occur in the presence of a moving surround. Such a decline would not be expected with higher spatial frequencies, since the visual response to these is more sustained in character and, therefore, less responsive to peripheral movement. Only 
slight influence of peripheral movement on steady or gradually presented test stimuli would also be expected, since these stimuli are not likely to produce on and off responses to stimulate mechanisms with transient-type time courses. A change in the shapes of the time-courses of mechanisms responsive to low spatial frequencies with peripheral movement should also be observable by obtaining histograms of reaction times to these spatial frequencies (e.g., Tolhurst, 1975). These should lose their onset and offset peaks and become unimodal with a single broad peak. In summary, the presence of peripheral motion is expected to inhibit the transient-type response, which should be revealed by a reduction in sensitivity to stimuli with high temporal and low spatial frequencies, and by a change in the shape of the distribution of reaction times to spatial frequencies. These possibilities were investigated in the following experiments.

\section{GENERAL METHODS}

\section{Apparatus.}

Stimulus patterns were generated by means of a "Picasso" CRT image generator (Innisfree Electronics) and displayed on a HP1310A VDU. The image generator concurrently produced two independently controlled grating stimuli with a frame frequency of $100 \mathrm{~Hz}$. Temporal parameters of the stimuli were controlled by 12-bit Dto-A outputs from a Microtrix microcomputer, which also controlled the experimental procedure. The luminance of all displays was $4.5 \mathrm{~cd} / \mathrm{m}^{2}$.

For each experiment, the display comprised a $13^{\circ}$ square field with a central $6^{\circ}$-diam circular area in which the test stimulus was presented. Binocular viewing with the natural pupil was used, at a distance on $114 \mathrm{~cm}$. The surrounding field was either a .6-contrast .65 -cycle/degree (cpd) square-wave grating or a sinusoidally flickering blank field. The square-wave grating was held steady or made to drift from left to right at a specified speed. The central area was blank except during the presentation of a stimulus. The test stimuli used in each of Experiments 1 to 4 were sinusoidal gratings of .3, $.5,1,2,4$, and $8 \mathrm{cpd}$. The lowest spatial frequencies were included because, although the number of cycles in the central field was very small (less than 2 in the case of $.3 \mathrm{cpd}$ ), the major interest of the experiment lay in observing the effects of peripheral movement on spatial frequencies in this region. Any effects of field size upon thresholds of the low spatial frequency gratings would have been constant across surround conditions.

\section{Procedure.}

Detection thresholds were measured by means of a modified "staircase" presentation generated by the computer. Subjects responded by entering " $Y$ " or " $N$," depending upon whether they could see (Y) or could not see $(N)$ the stimulus. It was also permissible to enter an "uncertain" response by entering another key, in which case the trial was repeated. A number of preliminary trials were initially given for each stimulus condition to establish the approximate threshold for that condition. This was followed by the experimental trials in which contrast was either raised or lowered in steps until there was a change in the subject's response from " $Y$ " to " $N$ " or vice versa. At this point (a reversal), the contrast of the stimulus was further raised or lowered by a random number of between 1 and 6 steps. Contrast was then adjusted in the opposite direction until the next reversal was obtained. The trials continued until a predetermined number of reversals had been completed for that condition. The threshold was taken to be the mean of these reversal points. Catch trials were randomly interspersed among the experimental trials in a ratio of approximately 1 to 3 .
In these, the contrast of the test stimulus was zero. If the subject responded "Y," an error message was given on the computer console, a bell sounded, and the sequence of trials returned to the beginning of the current reversal. This encouraged subjects to avoid careless responses.

\section{Subjects.}

Subjects included academic staff, research staff, and graduate students of the Department of Psychology.

\section{EXPERIMENT 1}

The effect of a moderate rate of peripheral movement on the detection thresholds of counterphase flickering and slowly pulsed sinusoidal gratings was investigated in the first experiment. The detection thresholds of the six test spatial frequencies were found in the presence and absence of peripheral movement.

\section{Method}

Thresholds were obtained for both a $5-\mathrm{Hz}$ counterphase flicker condition and a $.5-\mathrm{Hz}$ presentation. In each case, the test grating was presented for $1 \mathrm{sec}$, so that in the $.5-\mathrm{Hz}$ condition the stimulus had a cosine temporal envelope and the appearance of a grating being slowly turned on and off. There were two surround conditions, a steady and a moving condition. For the latter, the surrounding square-wave grating drifted at $2.5 \mathrm{cps}$.

There were eight subjects, each of whom participated under four experimental conditions. Each subject first underwent a series of practice trials in which the detection thresholds of each test spatial frequency were obtained with a steady surround. The threshold was the mean of four reversals. The experiment was a $2 \times 2 \times 6$ fully repeated measures design with 2 surround conditions, 2 test presentation conditions, and 6 spatial frequencies. The subjects varied considerably in the length of time required to complete the experiment. Consequently, the number of sessions differed over subjects, but most required 1-2 h, in one or two sessions. Separate two-way analyses of variance were conducted on the $.5-$ and $5-\mathrm{Hz}$ data.

\section{Results and Discussion}

The mean thresholds for the eight subjects are shown plotted as contrast sensitivity functions (CSFs) in Figure 1 . With the $5-\mathrm{Hz}$ flickering test stimuli, there was a slight decline in sensitivity to spatial frequencies lower than $2 \mathrm{cpd}$ in the movement condition as compared with the steady-surround condition. This difference was significant, as shown by the spatial frequency $\times$ surround interaction $[F(5,35)=5.41, p<.01]$. A decline in sensitivity was also observed with the $.5-\mathrm{Hz}$ data at .3 and $.5 \mathrm{cpd}$, although, in this case, the interaction was not significant $[F(5,35)=1.22, p>.05]$. It is also evident from Figure 1 that there was a large difference in the shapes of the CSFs obtained with .5- and 5-Hz flicker. Both the $.5-\mathrm{Hz}$ sets of data show a pronounced low-spatial-frequency attenuation, whereas this was virtually absent with the $5-\mathrm{Hz}$ condition.

The outcomes of this experiment indicate that although there was a significant decline in sensitivity to low spatial frequencies in the moving-surround $5-\mathrm{Hz}$ condition, this decline was slight compared with the pronounced lowfrequency attenuation obtained with the steady-surround $.5-\mathrm{Hz}$ condition. Thus, although the predicted decline was 


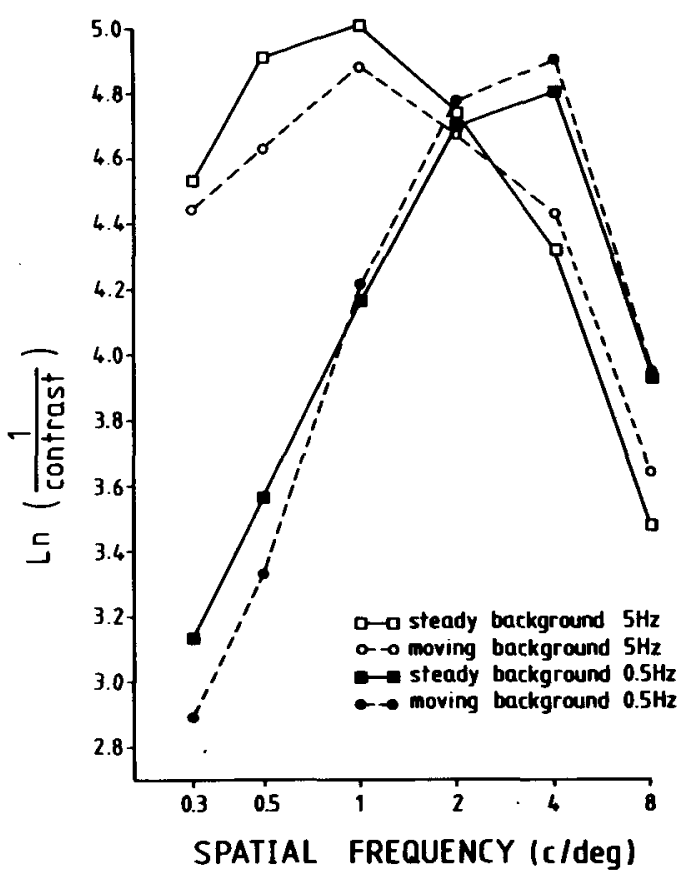

Figure 1. Contrast sensitivity functions for 1-sec presentations of 0.5- and 5-Hz counterphase flickering gratings obtained with steady surrounds and with surrounds moving at $2.5 \mathrm{cps}$. Each point is the mean of eight subjects' data.

observed, it was not as large as was expected if transient time-courses become more sustained in the presence of peripheral movement. It is, of course, conceivable that the rate of peripheral movement in this experiment was too slow to have a pronounced effect on the thresholds of the central test gratings.

Reaction time histograms were also obtained for .5-cpd gratings at $3 \mathrm{~dB}$ above threshold. There was no evidence of any change from the typical transient-type doublepeaked histogram observed with a steady surround to a more sustained-type single broad-peaked histogram with the moving surround. Thus, there was no clear evidence for a change from a transient-type response time-course at low spatial frequencies to a more sustained type with the surround moving at 2.5 cycles/sec (cps).

The greatest difference between the sensitivities to gratings with the steady and moving surrounds occurred at $.5 \mathrm{cpd}$, the frequency closest to that of the drifting surround. It has been demonstrated that a drifting grating produces illusory contours within a central blank area (Tynan \& Sekuler, 1975). Consequently, it could be argued that this result was due to a masking effect of illusory contours upon test gratings of similar spatial frequency, rather than to an effect of peripheral movement on the transient time-course.

The results of this experiment do not provide clear evidence for a change from transient to sustained-type activity in the presence of peripheral movement. The surround movement rate of $2.5 \mathrm{cps}$ was initially chosen because it was a relatively comfortable speed for subjects to observe. Since this speed might have been too slow to have a marked effect on the thresholds of low spatial frequencies, the experiment was repeated with a faster rate of peripheral movement, and with an additional control in which any masking effect due to illusory contours was eliminated.

\section{EXPERIMENT 2}

\section{Method}

Detection thresholds of 5 and $.5 \mathrm{~Hz}$ counterphase flickering gratings were again found. Thresholds were obtained with the peripheral steady square-wave grating, the same grating drifting at $6.8 \mathrm{cps}$, or a blank field flickering sinusoidally at $6.8 \mathrm{~Hz}$ with an amplitude of modulation of .6. The last condition was included to produce a condition in which the periphery was temporally modulated, but in which there was no possibility of inducing illusory contours which could mask the central test stimulus. There were three subjects. Each participated in all experimental conditions. Data for each combination of the flicker frequency of the central test grating and surround condition were obtained in a separate experimental session. The thresholds were the mean of eight reversals. The first eight reversals in a session were treated as practice and the data discarded. Separate two-way analyses of variance were carried out for the .5and $5-\mathrm{Hz}$ data.

\section{Results and Discussion}

The effects of the three surround conditions upon the detection thresholds of the .5- and $5-\mathrm{Hz}$ stimuli are illustrated in Figure 2.

With the 5-Hz counterphase flickering stimuli, the steady-surround CSF peaks at $1 \mathrm{cpd}$ with a slight reduction in sensitivity at the lower spatial frequencies. This is the characteristic shape of the CSF obtained when motion-sensitive mechanisms are stimulated (Kulikowski \& Tolhurst, 1973). In contrast to this, the 5-Hz CSFs with both peripheral movement and flicker show a pronounced low-frequency attenuation. With both types of surround, the drop in sensitivity to .3-, .5-, and 1-cpd gratings is substantial. At $2 \mathrm{cpd}$, there is a smaller drop, but at 4

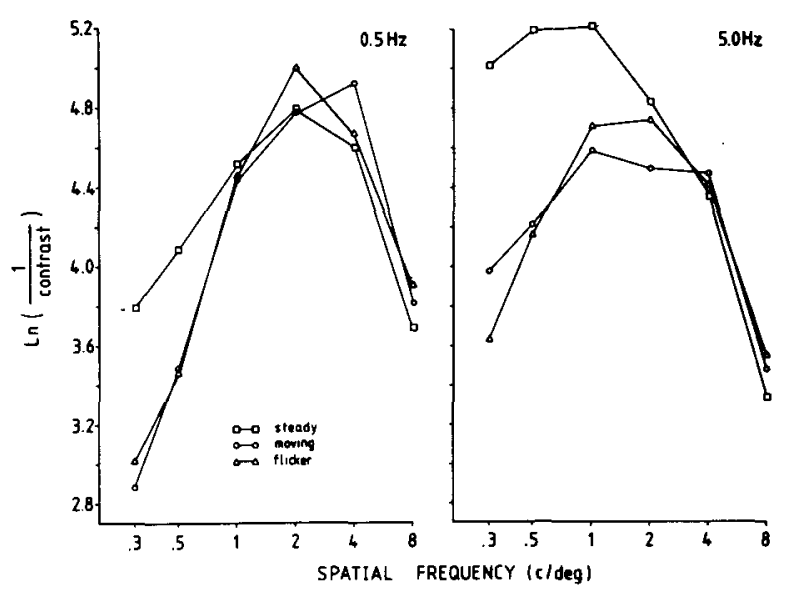

Figure 2. Contrast sensitivity functions for $1-\sec$ presentations of 0.5- and $5-\mathrm{Hz}$ counterphase flickering gratings obtained with steady surrounds and with 6.8-Hz flickering and moving surrounds. Each point is averaged over three subjects. 
and $8 \mathrm{cpd}$, there is a slight increase in sensitivity above that of the CSF obtained with the steady surround. This difference in the shapes of the CSFs is statistically significant, as indicated by the surround $X$ spatial frequency interaction $[F(10,20)=10.63, p<.01]$.

The CSFs obtained with both moving and flickering surrounds are similar in shape. This indicates that the temporal modulation of the surround probably accounts for the reduced sensitivity under both surround conditions. The possibility that masking by illusory contours is a major source of the sensitivity difference between the steady and moving surround conditions thus appears to be eliminated.

The moving- and flickering-surround 5- $\mathrm{Hz}$ CSFs are similar in shape to the steady-surround .5-Hz CSF. This indicates that the mechanisms operating at $5 \mathrm{~Hz}$ with the temporally modulated periphery may be similar to those operating at threshold in the $.5-\mathrm{Hz}$ steady surround condition. These data consequently provide clear evidence for a suppression of transient-type activity when the test stimulus is surrounded by 6.8-cpd temporal modulation, whether a moving vertical grating or a sinusoidally flickering blank field.

Because there were no high temporal frequencies present in the .5-Hz stimuli, mechanisms with a transient timecourse were not expected to be stimulated. The steadysurround condition exhibited the pronounced low-spatialfrequency attenuation normally observed under conditions thought to stimulate mechanisms with a sustained timecourse. Since temporal modulation of the periphery was not expected to affect these mechanisms, no further effect on the thresholds of the $.5-\mathrm{Hz}$ low-frequency gratings was anticipated. It is obvious from Figure 2, however, that a considerable decrease in sensitivity occurred at .3 and $.5 \mathrm{cpd}$ with both moving and flickering surrounds. This decrease was significant $[\mathrm{F}(10,20)=5.86$, $\mathrm{p}<.01]$. Again, the CSFs obtained with the two temporally modulated surrounds were similar in shape, and again there was a slight rise in threshold with the 4- and 8-cpd stimuli. This effect of peripheral movement on the $.5-\mathrm{Hz}$ stimulus will be considered further in more detail below.

\section{EXPERIMENT 3}

Briefly pulsed stimuli do not produce contrast sensitivity functions with pronounced low-frequency attenuations (e.g., Legge, 1978), and it is probable that this type of stimulus vigorously stimulates mechanisms with transient time-courses. Peripheral movement may thus be expected to influence sensitivity to briefly pulsed sinuoidal gratings at low spatial frequencies. CSFs to brief pulses presented along with peripheral movement may thus show a pronounced low-frequency attenuation as compared with a similar CSF obtained with a steady surround. The third experiment was designed to test this hypothesis, and to investigate any effects of variation in the orientation and direction of movement of the peripheral grating. An ad- ditional surround condition in which the grating was horizontal and moved upwards at a rate of $6.8 \mathrm{~Hz}$ was employed. The test stimulus remained vertical. Any illusory contours induced by this surround were thus at right angles to the test stimulus and would have reduced any masking effect of these on the test grating.

\section{Method}

There were four surround conditions: a steady vertical grating, a vertical grating moving from left to right at $6.8 \mathrm{cps}$, a horizontal grating moving upwards at $6.8 \mathrm{cps}$, and a sinusoidally flickering field. Detection thresholds of 60 -msec pulses of the six test spatial frequencies were obtained for the three subjects employed in the previous experiment.

\section{Results and Discussion}

The data are shown in Figure 3. The CSF measured with the steady surround peaks between 1 and $2 \mathrm{cpd}$, with a slight low-frequency falloff. This is similar to the result for Experiment 2. In comparison, the CSFs obtained with the vertical moving and the flickering surrounds both showed a pronounced low-spatial-frequency attenuation. There was also a slight rise in sensitivity at 4 and $8 \mathrm{cpd}$ under these conditions. This difference in the shapes of the CSFs was significant, as indicated by a significant surround $\times$ spatial frequency interaction $[\mathrm{F}(15,30)=5.38$, $\mathrm{p}<.01$ ]. These data are essentially similar to the $5-\mathrm{Hz}$ counterphase flicker data of Experiment 2, and again in-

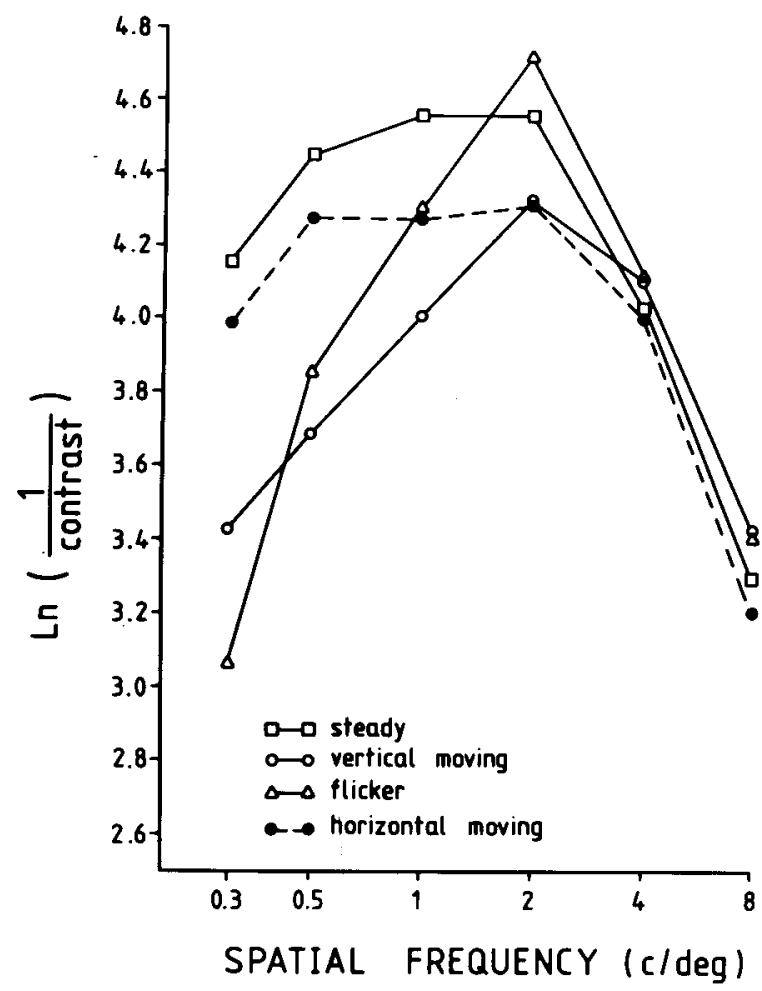

Figure 3. Contrast sensitivity functions for 60 -msec pulsed gratings obtained with steady and with $6.8-\mathrm{Hz}$ flickering vertical and horizontal moving surrounds. Each point is the mean of three subjects' data. 
dicate that there is a suppression of transient-type activity at low spatial frequencies with flickering or vertical moving surrounds.

The CSF obtained with the horizontal surround drifting upwards does not show the same degree of lowfrequency attenuation as those obtained with the other temporally modulated surrounds. There is a significant interaction between surround and frequency in a separate comparison of the horizontal with the vertical moving-surround condition $[\mathrm{F}(5,10)=4.23, \mathrm{p}<.05]$ but no interaction when the horizontal moving condition is compared with the steady condition $[\mathrm{F}(5,10)=0.15, \mathrm{p}>$ $.05]$. The CSF obtained with the horizontal surround is consequently more similar in shape to that of the steady surround than to the other temporally modulated surrounds. This effect of the orientation of the moving surround on the shape of the CSF was unexpected. Consequently, it was investigated in more detail in the fourth experiment.

\section{EXPERIMENT 4}

In the third experiment, the test stimulus was a 60 -msec pulsed grating. The aim of this experiment was to further investigate the effect of the orientation of the surrounding moving grating, using $5-\mathrm{Hz}$ counterphase flickering test stimuli.

\section{Method}

Contrast sensitivity measured in the presence of a vertical surround moving left-right was compared with that obtained with a horizontal surround moving upwards. An additional vertical steadysurround condition was included as a control. To produce a horizontal surround in the presence of the vertical test stimulus, the frame interleaving module of the CRT image generator was used. Since the use of this module caused a slight flicker in the display at the onset and offset of the test stimuli and the contrast settings also required readjusting, all stimuli with the vertical surround (including the steady control) were similarly presented with the frame interleaving module. The spatial frequency, contrast, and temporal frequency of the motion of the surround were the same as in the earlier experiments. Data for each surround condition were obtained in separate sessions. There were four subjects.

\section{Results and Discussion}

The contrast sensitivity functions obtained with the moving surrounds show a pronounced low-spatial-frequency attenuation, as compared with that obtained with the steady surround (Figure 4). This difference between the shapes of the functions is significant, as shown by the significant surround $x$ frequency interaction in the analysis of variance $[\mathrm{F}(10,30)=7.49, \mathrm{p}<.01]$. This result confirms that of Experiment 2, in which a pronounced lowfrequency attenuation to the CSF was similarly obtained in the presence of the vertical drifting surround. However, there was one minor discrepancy between the results of the two experiments. Sensitivity to the 4- and 8-cpd test stimuli with the steady surround was always higher than that obtained in the presence of the vertical moving surround in Experiment 4, in contrast to the result in Ex-

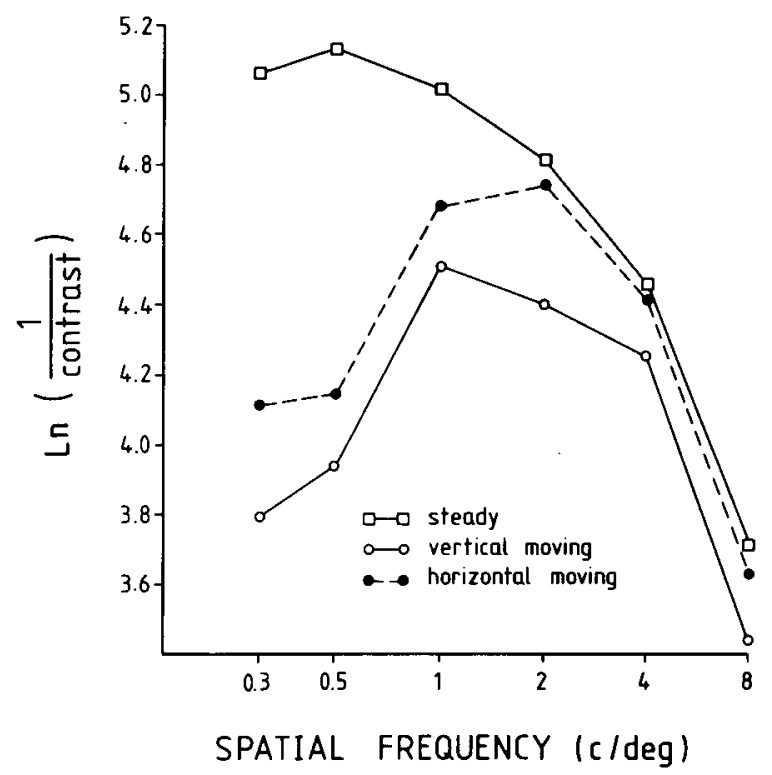

Figure 4. Contrast sensitivity functions for 1-sec presentations of 5-Hz counterphase flickering gratings in the presence of steady vertical and horizontal moving square-wave grating surrounds.

periment 2. The reason for this discrepancy is not clear; it may conceivably be related to the slightly different experimental arrangement used in Experiment 4, with the frame interleaving module employed in all three surround conditions.

To investigate differences in the effects of vertical and horizontal moving surrounds, the data were compared separately. As is evident in Figure 4, the shapes of the two functions are similar. This is confirmed by the lack of any surround $x$ frequency interaction in the vertical vs. horizontal surround comparison $[F(5,15)=0.36$, $p>.05]$. A comparison of the horizontal moving condition with the steady condition, however, indicates that there is a significant frequency $\times$ surround interaction $[F(1,3)=44.11, p<.01]$. Thus, the presence of a horizontal surround produces a significant attenuation to the CSF at low spatial frequencies. This does not differ significantly from the attenuation produced by the vertical moving surround. With $5-\mathrm{Hz}$ flickering test stimuli, the orientation of the moving surround consequently does not have a marked effect on the reduction in sensitivity to low spatial frequencies produced by peripheral movement.

The discrepancy between these outcomes and those of the previous experiment may be due to the difference in the temporal presentation of the test stimuli. However, in both experiments, it is evident that the degree of attenuation produced at low spatial frequencies is greater with the vertical moving surround than with the horizontal surround. Consequently, it appears that a surrounding grating moving in a direction orthogonal to the test stimulus considerably reduces sensitivity to low spatial frequencies, but to a slightly lesser extent than a surround 


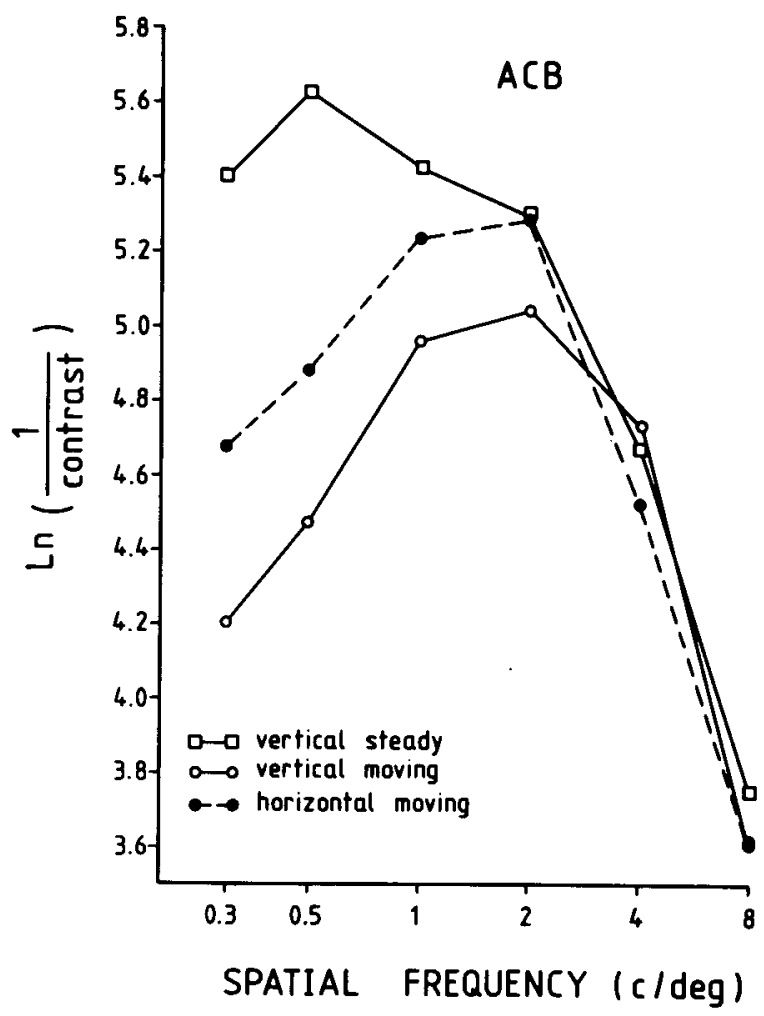

Figure 5. Contrast sensitivity functions for 1-sec presentations of 5-Hz moving gratings with steady and with $6.8-\mathrm{Hz}$ vertical and horizontal moving surrounds (Subject A.C.B.).

of the same orientation as the test stimulus. This was also found to be the case in an additional observation, by the author, using a 1-sec presentation of a $5-\mathrm{Hz}$ drifting test stimulus. Clear low spatial frequency attenuation was observed with the horizontal moving surround (see Figure 5), but the degree of attenuation was less than that with the vertical surround.

\section{EXPERIMENT 5}

Tolhurst (1975) showed, by means of reaction time histograms, that the visual response to low spatial frequencies is transient, responding to the onsets and offsets of stimuli. Reaction times were collected to low-contrast pulsed presentations of the gratings. The distribution of reaction times to low spatial frequencies comprised double-peaked histograms with the positions of the peaks corresponding to the stimulus onset and offset. Histograms obtained with higher frequency stimuli, however, had a single broad peak indicating that the stimulus might become detectable at any time during its presentation, and hence indicating a sustained-type response. This method has since been used by others to investigate the timecourse of the visual response obtained under varying experimental conditions (e.g., Breitmeyer, Levi, \& Harwerth, 1981; Smith, Harwerth, Levi, \& Boltz, 1982).
If peripheral movement and flicker cause the timecourse of the visual response to low spatial frequencies to shift from predominantly transient to predominantly sustained, the stimulus should now become detectable at any time during its presentation. Reaction-time histograms obtained with moving and flickering surrounds should consequently become single-peaked, as compared with a double-peaked histogram in the steady-surround condition. Reaction time histograms were obtained for a .5-cpd 500 -msec pulse, using the same three surround conditions as employed in Experiment 2-a steady vertical grating, this grating drifting at $6.8 \mathrm{cps}$, and the sinusoidally flickering field.

\section{Method}

There were two subjects, each of whom completed one experimental session under each condition. In each session, the detection threshold of the $500-\mathrm{msec}$ stimulus was first measured with the specific surround condition for that session. Each threshold was the mean of 16 reversals. Ten practice and 200 experimental reaction time trials were then presented at $3 \mathrm{~dB}$ above threshold. Each trial was initiated by pressing the space bar of the computer keyboard. This was followed by a bell signal and a random delay of between 1 and $2 \mathrm{sec}$ before stimulus onset. The subject responded by pressing any key on the keyboard (except ESC). If a response did not occur by $1,000 \mathrm{msec}$ after stimulus onset, the trial was disregarded, as was any trial in which a response occurred prior to stimulus presentation.

\section{Results and Discussion}

The reaction time histograms for each subject are shown in Figure 6. Both subjects produced double-peaked histograms in the steady surround condition, with the peak as-

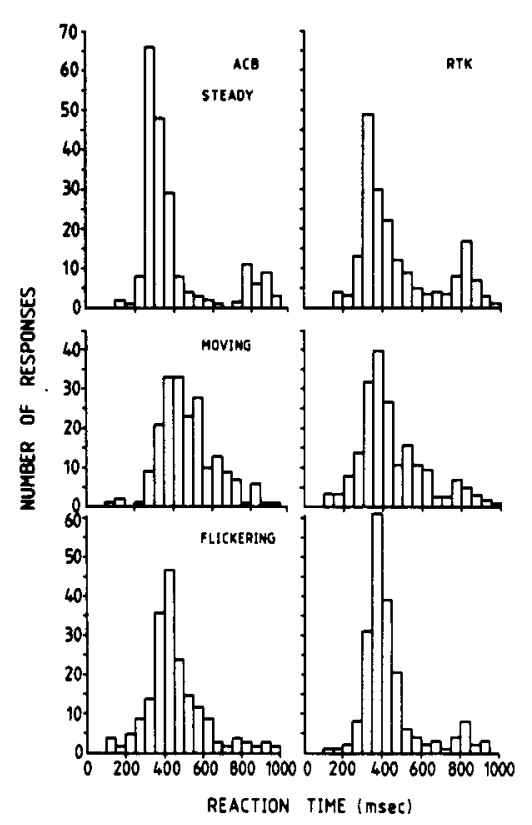

Figure 6. Histograms of the distributions of 200 reaction times obtained with steady and with $6.8-\mathrm{Hz}$ flickering and moving surrounds for two subjects (A.C.B. and R.T.K.). 
sociated with stimulus onset more pronounced than the offset peak for both subjects. With the moving and flickering surrounds, the shapes of the histograms became less characteristic of the transient time-course, with a single, broader peak predominating (although this is not as strong in the flicker surround data of R.T.K.). These data provide further evidence for a suppression of the activity of mechanisms responding with a transient time-course in the presence of temporal modulation in the peripheral visual field.

\section{GENERAL DISCUSSION}

The five experiments provide evidence that the activity of the mechanisms responsible for the detection of sinusoidal gratings is affected by temporal modulation of the field surrounding the test stimulus. A comparison of the results of Experiments 1 and 2 reveals that the reduction in sensitivity to low spatial frequencies is dependent upon the frequency of temporal modulation of the periphery; the reduction is more pronounced with a 6.8-cps moving surround than with a 2.5 -cps surround.

In Experiments 1, 2, and 3, the effect of a vertical moving grating or a flickering-field surround was to decrease sensitivity to flickering or briefly pulsed sinusoidal gratings with spatial frequencies less than 2 cpd. In each of these experiments, there was also a very slight increase in sensitivity at 4 and $8 \mathrm{cpd}$. This increase in sensitivity was not, however, observed in Experiment 4. The data are similar to those recently reported by Green (1983), who showed that a flickering uniform field surrounding a test field reduces the detection thresholds of gratings moving within the central field. In Green's experiments, as in those described here, a pronounced attenuation was observed with spatial frequencies lower than 2 or $3 \mathrm{cpd}$ in the presence of temporally modulated surrounds. Green observed that test grating drift frequencies of as low as $.6 \mathrm{~Hz}$ were sufficient to produce a threshold elevation with the flickering surround, but greater elevations were produced with drift frequencies of $1.2 \mathrm{~Hz}$ and above. No change in threshold was observed with a .54-cpd test stimulus slowly turned on and off with a cosine temporal envelope. This differs from the results of Experiment 2, in which .3- and .5-cpd stimuli presented with a cosine temporal envelope were found to exhibit marked sensitivity differences in the presence of the moving or flickering surrounds.

The results of Experiments 1 to 3 also resemble those of Breitmeyer et al. (1981), in which a similar low-spatialfrequency attenuation was observed in the presence of a 6-Hz counterphase flickering background uniform field. A low-frequency attenuation was also observed when a pattern detection threshold was employed, but in this instance problems in maintaining criterion may account for the data (Lennie, 1980).

It is possible that the $.5-\mathrm{Hz}$ stimulus in Experiment 2 was brief enough to produce a temporal change at its on- set and offset sufficient to stimulate on- and off-activity. This would then allow for a further reduction in sensitivity in the presence of the temporally modulated surrounds. Green (1983) did not mention the total duration of his cosine temporal envelope .54-cpd stimulus, and it is thus possible that the two stimuli differed sufficiently in duration to account for the varying results of the two experiments in this regard. Alternatively, there may be only one type of channel sensitive to low spatial frequencies. Peripheral movement may modify the characteristics of this channel's response in such a way as to influence sensitivity to both temporally modulated and steady stimuli. This possibility gains support from the recent failure to find evidence for differences between the thresholds to pattern and form when differences due to associated criterion shifts are eliminated (e.g., Burbeck, 1981; Lennie, 1980).

The results of the experiments reported here indicate that peripheral movement and flicker alter the temporal characteristics of mechanisms responsive to low spatial frequencies. This effect may perhaps be interpreted by assuming the existence of a network of retinal (and higher?) subunits which interacts with the mechanisms determining detection threshold. Evidence for the existence of such a network in the cat is accumulating (e.g., Shapley \& Victor, 1978), and recent work on the "far-out jerk effect" may indicate the presence of a similar mechanism in man (e.g., Valberg \& Breitmeyer, 1980). Temporal modulation of the periphery may stimulate and possibly saturate the activity of this network. This activity may then influence the nonlinear mechanism producing the "transient" time-course to low spatial frequencies, reducing sensitivity to temporally modulated gratings of these frequencies. A number of experiments have been reported which have been interpreted as evidence for the adaptation or masking of transient mechanisms (Breitmeyer et al., 1981). These could alternatively be explained on the basis of a temporary or longer term saturation of this hypothesized nonlinear network with its consequent inhibition of the transient component of a response, making the system less responsive to further temporal change.

In summary, these experiments have demonstrated that the response of mechanisms sensitive to low spatial frequencies is modifiable by movement and flicker in the peripheral visual field. This effect is explained in terms of interactions between a nonlinear network and those mechanisms that are sensitive to gratings at threshold. It is thus reasonable to suppose that activity within the hypothesized network inactivates or saturates the mechanism that normally gives rise to the "transient" time-course of the low-spatial-frequency channels.

\section{REFERENCES}

Breitmeyer, B., Levi, D. M., \& Harwerth, R. S. (1981). Flicker masking in spatial vision. Vision Research, 21, 1377-1385.

BurbeCK, C. A. (1981). Criterion-free pattern and flicker thresholds. Journal of the Optical Society of America, 71, 1343-1350. 
Derrington, A. M., Lennie, P., \& Wright, M. J. (1979). The mechanisms of peripherally evoked responses in retinal ganglion cells. Journal of Physiology (London), 289, 299-310.

GreEN, M. (1983). Visual masking by flickering surrounds. Vision Research, 23, 735-744.

HochsteIN, S., \& SHAPLEY, R. M. (1976). Linear and nonlinear spatial subunits in Y cat retinal ganglion cells. Journal of Physiology (London), 262, 265-284.

KRUGer, J. (1980). The shift-effect enhances X-and suppresses Y-type response characteristics of cat retinal ganglion cells. Brain Research, 201, 71-84.

KRUGER, J. (1981). The difference between X-and Y-type responses in ganglion cells of the cat's retina. Vision Research, 21, 1685-1687.

KULukowski, J. J., \& TolHURST, D. J. (1973). Psychophysical evidence for sustained and transient detectors in human vision. Joumal of Physiology (London), 232, 149-162.

LEGGE, G. E. (1978). Sustained and transient mechanisms in human vision: Temporal and spatial properties. Vision Research, 18, 69-81.

LENNIE, P. (1980). Perceptual signs of parallel pathways. Philosophical Transactions of the Royal Society, London, Series B, 290, 23-37.

Mitov, D., Vassilev, A., \& Manahilov, V. (1981). Transient and sustained masking. Perception and Psychophysics, 30, 205-210.
Robson, J. G. (1966). Spatial and temporal contrast sensitivity functions of the visual system. Journal of the Optical Society of America, 56, 1176-1186.

Shapley, R. M., Victor, J. D. (1978). The effect of contrast on the transfer properties of cat retinal ganglion cells. Journal of Physiology (London), 285, 275-298.

Smith, E. L., Harwerth, R. S., Levi, D. M., \& Boltz, R. L. (1982). Contrast increment thresholds of thesus monkeys. Vision Research, 22, 1153-1160.

TOLHURST, D. J. (1975). Reaction times in the detection of gratings by human observers: A probabalistic mechanism. Vision Research, $15,1143-1149$.

Tynan, P., \& Sekuler, P. (1975). Moving visual phantoms: A new completion effect. Science, 188, 951-952.

VAlBerg, A., \& Breitmeyer, B. (1980). The lateral effect of oscillation of peripheral luminance gratings: Test of various hypotheses. $V i$ sion Research, 20, 789-798.

(Manuscript received July 26, 1984; accepted for publication February 11, 1985.) 